\title{
Multi-reference approach to the computation of double core-hole spectra
}

\author{
Tenorio, Bruno Nunes Cabral; Decleva, Piero; Coriani, Sonia
}

Published in:

Journal of Chemical Physics

Link to article, DOI:

$10.1063 / 5.0062130$

Publication date:

2021

Document Version

Peer reviewed version

Link back to DTU Orbit

Citation (APA):

Tenorio, B. N. C., Decleva, P., \& Coriani, S. (2021). Multi-reference approach to the computation of double corehole spectra. Journal of Chemical Physics, 155(13), [131101]. https://doi.org/10.1063/5.0062130

\section{General rights}

Copyright and moral rights for the publications made accessible in the public portal are retained by the authors and/or other copyright owners and it is a condition of accessing publications that users recognise and abide by the legal requirements associated with these rights.

- Users may download and print one copy of any publication from the public portal for the purpose of private study or research.

- You may not further distribute the material or use it for any profit-making activity or commercial gain

- You may freely distribute the URL identifying the publication in the public portal

If you believe that this document breaches copyright please contact us providing details, and we will remove access to the work immediately and investigate your claim 


\title{
Communication: Multi-reference Approach to the Computation of Double-Core-Hole Spectra
}

\author{
Bruno Nunes Cabral Tenorio, ${ }^{1, a)}$ Piero Decleva, ${ }^{2}$ and Sonia Coriani ${ }^{3, b)}$ \\ 1) DTU Chemistry - Department of Chemistry, Technical University of Denmark, \\ Kemitorvet Bldg 207, 2800 Kongens Lyngby, Denmark \\ ${ }^{2)}$ Istituto Officina dei Materiali IOM-CNR and Dipartimento di Scienze \\ Chimiche e Farmaceutiche, Università degli Studi di Trieste, I-34121 Trieste, \\ Italy \\ 3) DTU Chemistry - Department of Chemistry, Technical University of Denmark, \\ Kemitorvet Bldg 207, 2800 Kongens Lyngby, Denmark, \\ and NTNU - Norwegian University of Science and Technology, N-7491 Trondheim, \\ Norway
}

(Dated: 22 September 2021)

Double-Core Hole (DCH) states of small molecules are assessed with the restricted active space self-consistent field (RASSCF) and multi-state restricted active space perturbation theory of second order (MS-RASPT2) approximations. To ensure an unbiased description of the relaxation and correlation effects on the DCH states, the neutral ground state and DCH wave functions are optimized separately, whereas the spectral intensities are computed with a biorthonormalized set of molecular orbitals within the state-interaction (SI) approximation. Accurate shake-up satellites binding energies and intensities of double-core-ionized states $\left(K^{-2}\right)$ are obtained for $\mathrm{H}_{2} \mathrm{O}$, $\mathrm{N}_{2}$, $\mathrm{CO}$ and $\mathrm{C}_{2} \mathrm{H}_{2 n}(n=1-3)$. The results are analyzed in details and show excellent agreement with recent theoretical and experimental data. The $K^{-2}$ shake-up spectra of $\mathrm{H}_{2} \mathrm{O}$ and the $\mathrm{C}_{2} \mathrm{H}_{2 n}$ molecules are here completely characterized for the first time.

\footnotetext{
a)Electronic mail: brncat@dtu.dk

b)Electronic mail: soco@kemi.dtu.dk
} 
With the advent of the newest generation of x-ray sources, double core-shell photoionization spectroscopy has been reported with unprecedented resolution either from two-photon photoelectron spectroscopy using x-ray free electron laser (X-FEL) radiation ${ }^{1-6}$ or with single x-ray photon at synchrotron facilities. ${ }^{7-16}$ Single-site double core hole (ssDCH or $K^{-2}$ ) spectroscopy has gained special attention due to, among others, its chemical specificity and enhanced sensitivity to chemical environment when compared to single core hole (SCH) spectroscopy. ${ }^{2}$ The higher sensitivity of ssDCH spectroscopy to the chemical environment has been demonstrated, for example, in the $K^{-2}$ spectra of the $\mathrm{C}_{2} \mathrm{H}_{2 n}(n=1-3)$ series, where the shift in binding energy of the first peak of $\mathrm{C}_{2} \mathrm{H}_{4}$ relative to $\mathrm{C}_{2} \mathrm{H}_{2}$ was measured to be $\Delta E_{\mathrm{C}_{2} \mathrm{H}_{4} / \mathrm{C}_{2} \mathrm{H}_{2}}=-2.1 \mathrm{eV},{ }^{17}$ versus the value $\Delta E_{\mathrm{C}_{2} \mathrm{H}_{4} / \mathrm{C}_{2} \mathrm{H}_{2}}=-0.5 \mathrm{eV}$ obtained from the $\mathrm{SCH}$ spectra. ${ }^{18}$ Its characteristics upraise ssDCH spectroscopy as one of the most promising techniques to chemical analysis. However, in contrast to $\mathrm{SCH}$ spectroscopy, ${ }^{19-22}$ it has been widely demonstrated that some of the most important features of a typical DCH spectrum arise from strong shake-up satellite states,${ }^{14,17,23}$ which stem from excitation of one or more outer electrons in combination with the photoionization of two core electrons located on the same atom. Shake-up satellite states have intrinsic multielectronic character and are

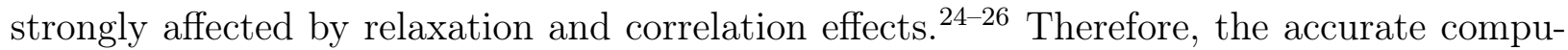
tation of ssDCH spectra depends on the appropriateness of the computational method to be used in dealing with these effects.

Relaxation and correlation effects are in principle different concepts. Electronic relaxation is regarded as the shrinking of the electron cloud in response to the formation of a deep core hole. Correlation, on the other hand, is a theoretical concept associated with deviations from the independent particle picture. Put in simple terms, correlation can be assessed by the difference between a mean field Hartree-Fock (HF) calculation and the full configuration-interaction (Full-CI) result in the limit that the basis set approaches completeness. It may be worth recalling that even relaxation can be considered a correlation effect, and not separately treated. Indeed, in several many-body schemes aiming at describing ionization, like algebraic-diagrammatic construction $(\mathrm{ADC})^{27-29}$ or equation-of-motion coupled cluster (EOM-CC) theory, ${ }^{30-32}$ relaxation is treated as pure correlation. In the context of ssDCH spectra, a few computational strategies have been proposed. Pioneering studies by Cederbaum et al..$^{33-35}$ have shown the extraordinary sensitivity of ssDCH states even before any experimental technique could allow their observation with the resolution level that we 
have today. A seminal work by Agren and Jensen ${ }^{36}$ described the relaxation and correlation contributions to double core ionization energies, and how one could compute them using multi-configuration self-consistent field (MCSCF) wave-functions, ${ }^{37-39}$ in particular, the restricted active space (RAS) approach. ${ }^{38}$ RAS constitutes a threefold division of the correlation orbital space, denoted RAS1, RAS2 and RAS3.

A number of theoretical works aimed at double core hole states have been reported over the past few years, employing different levels of theory, ranging from time-dependent density functional theory (TD-DFT), ${ }^{40,41} \mathrm{DFT},{ }^{6,42-44}$ and $\triangle \mathrm{SCF},{ }^{6,45}$ to MCSCF,${ }^{6,42-44,46,47}$ $\Delta$ Coupled Cluster $(\Delta \mathrm{CC}),{ }^{48,49}$ and many-body Green's function. ${ }^{45,50,51}$ Most of these works, however, were limited to the computation of double core ionization potentials. A previous attempt to compute the ssDCH spectra of the $\mathrm{CO}$ molecule using the RAS approach was presented by Tashiro et al., ${ }^{52}$ but a too simple approximation on the transition amplitudes prevented the authors from reproducing the spectra at a satisfactory level.

It was only recently that an accurate method to compute ssDCH binding energies as well as spectral strengths was proposed by Ferté et al. and applied to the CO molecule. ${ }^{23}$ The method combines a CI approach using perturbative selection (CIPSI) ${ }^{53,54}$ with the computation of transition amplitudes using two sets of non-orthogonal molecular orbitals. One set was obtained from a restricted HF calculation on the ground state configuration; the second (relaxed) set was generated from a non-Aufbau SCF calculation with two electrons removed from the core orbital. At this approximation level, dubbed NOTA + CIPSI by the authors, the oxygen double core edge spectrum of carbon monoxide was obtained in excellent agreement with the experimental data.

In the present work, we propose a multi-reference protocol to compute ssDCH spectra using the restricted active space self-consistent field (RASSCF) approach ${ }^{38}$ as well as multistate restricted active space perturbation theory of second order (MS-RASPT2). ${ }^{55-58}$ In our approach, the transition amplitudes are determined within the sudden approximation using a biorthonormalized set of molecular orbitals, where the ground state and the double core hole states are obtained from different RASSCF calculations. This ensures an unbiased representation of the initial and final states and an appropriate description of the relaxation and correlation effects associated with the shake-up satellites of a ssDCH spectrum. Moreover, the use of a biorthonormalized orbital set ${ }^{59}$ significantly simplifies the computation of matrix elements between different states, reducing the computational cost of such calculations. 
Accurate and computationally efficient ssDCH multi-reference calculations on molecules with equivalent atoms require localization of the core orbitals. ${ }^{36}$ Delocalized core orbitals are typically localized with one of the well-known Boy's ${ }^{60}$ or Pipek-Mezey's ${ }^{61}$ localization procedures. However, application of these methods has the disadvantage of breaking the point group symmetry, which is a complicating factor for the analysis of excited states. Besides, it is in general computationally more efficient if one can take advantage of point group symmetry in the calculation of a large number of excited states. Therefore, we here propose to use Cholesky localized orbitals instead of Boys' or Pipek-Mezey's orbitals. The Cholesky molecular orbitals are defined through the Cholesky decomposition of the oneelectron density matrix, and inherit locality from the sparsity of the density matrix. ${ }^{62}$ As discussed later, Cholesky molecular orbitals allow us to retain some degree of point-group symmetry in the calculations.

In detail, we consider a molecule with $N$ electrons initially in its ground state $\left|\Psi_{i}^{N}\right\rangle$. Following the single-channel approximation, we represent the wave-function $\left|\Psi_{f}^{N}\right\rangle$ of the final state where two electrons have been ejected into the continuum as an antisymmetrized product of the wave-function of the molecular (bound) $(N-2)$ double-core-hole final state, $\left|\Psi_{f}^{N-2}\right\rangle$, and the continuum wave-function, $\left|\psi^{e l}\left(\overrightarrow{k_{1}}, \overrightarrow{k_{2}}\right)\right\rangle$, of the two photoelectrons with asymptotic momenta $\overrightarrow{k_{1}}$ and $\overrightarrow{k_{2}}$,

$$
\left|\Psi_{f}^{N}\right\rangle=\hat{\mathcal{A}}(N)\left|\Psi_{f}^{N-2} \psi^{e l}\left(\overrightarrow{k_{1}}, \overrightarrow{k_{2}}\right)\right\rangle
$$

where $\hat{\mathcal{A}}(N)$ is the $N$-electron antisymmetrizer operator.

Within the dipole approximation, the differential cross-section connecting the initial state and the final state wave-function - in atomic units and in the length gauge - is given by

$$
\frac{d^{2} \sigma}{d \overrightarrow{k_{1}} d \overrightarrow{k_{2}}}=4 \pi^{2} \alpha \omega\left|\vec{D}_{i f}\right|^{2}
$$

where $\vec{D}_{i f}=\left\langle\Psi_{i}^{N}|\vec{\mu}| \Psi_{f}^{N}\right\rangle$ is the dipole transition moment, $\alpha$ is the fine-structure constant, and $\omega$ is the energy of the incident photon.

In analogy with the Dyson orbital formulation for single ionization, ${ }^{63,64}$ the full dipole element in Eq. (2) can be approximated by the term involving a dipole transition between a two-particle Dyson function, defined by the amplitudes

$$
\gamma_{k l}^{f i}=\left\langle\Psi_{f}^{N-2}\left|a_{k} a_{l}\right| \Psi_{i}^{N}\right\rangle
$$


and the two-particle continuum function in Eq. (1). For double core ionization, the only significant amplitudes are those involving the core orbitals $1 s$. Moreover, especially at high energy, the continuum function in Eq. (1) is rather independent on the specific final double core hole ionic state, so that the dipole contribution will be essentially constant over the manifold of final states. Therefore the relative intensities, or spectral strengths, associated with a peak in the ssDCH spectrum can be estimated as

$$
\begin{aligned}
\left|T_{i \rightarrow f}\right|^{2} & \propto\left|\left\langle\Psi_{f}^{N-2}\left|a_{1 s}^{\alpha} a_{1 s}^{\beta}\right| \Psi_{i}^{N}\right\rangle\right|^{2} \\
& =\left|\left\langle\Psi_{A}^{N-2} \mid \Psi_{B}^{N-2}\right\rangle\right|^{2}
\end{aligned}
$$

where $a_{1 s}^{\sigma}$ is the second-quantization annihilation operator of the electron of spin $\sigma$ in orbital $1 s$.

As highlighted by the second equality in Eq. (4), the spectral strengths correspond to overlap matrix elements between two $(N-2)$ electron wave functions. By using a biorthonormalized set of molecular orbitals in Eq. (4), as done in the Restricted Active Space - State Interaction (RASSI) method, ${ }^{59,65}$ the computation of these matrix elements is very efficient and straightforward, as it would be to compute the overlaps with a common orthonormal molecular orbital basis.

The RASSI method has been used to compute matrix elements over distinct (biorthonormal) molecular orbital (MO) sets of inner-shell states with great success. ${ }^{66-68}$ To briefly recapitulate the attributes of the RASSI method, ${ }^{59,65}$ let us first write the matrix elements between two distinct optimized wave functions $\Psi_{A}$ and $\Psi_{B}$ described by a CI expansion of Slater determinant functions (SDF) or spin-adapted configuration state functions (CSF), $\Phi^{A}$ and $\Phi^{B}$, constructed from the sets of molecular orbitals $\left\{\phi^{A}\right\}$ and $\left\{\phi^{B}\right\} \quad\left(\Psi_{A}\right.$ and $\Psi_{B}$ can be generalized to the case of CASSCF or mixed CASPT2 wave-functions)

$$
\begin{array}{ll}
\Psi_{A}=\sum_{\mu} C_{\mu}^{A} \Phi_{\mu}^{A}, & \Phi_{\mu}^{A}=a_{p}^{\dagger} \ldots a_{s}^{\dagger}|\mathrm{vac}\rangle \\
\Psi_{B}=\sum_{\nu} C_{\nu}^{B} \Phi_{\nu}^{B}, & \Phi_{\nu}^{B}=a_{q}^{\dagger} \ldots a_{r}^{\dagger}|\mathrm{vac}\rangle
\end{array}
$$

where $\mu$ is a lists of occupied orbitals $(p \ldots s)$, and similarly for $\nu$.

The overlap matrix between the wave functions can be written as

$$
\left\langle\Psi_{A} \mid \Psi_{B}\right\rangle=\sum_{\mu \nu} C_{\mu}^{A} C_{\nu}^{B} S_{\mu \nu}
$$


with $S_{\mu \nu}$ as the overlap matrix elements between two SDF, $S_{\mu \nu}=\left\langle\Phi_{\mu}^{A} \mid \Phi_{\nu}^{B}\right\rangle$. The overlap matrix $S$ becomes the unit matrix provided that the two sets of spin-orbitals are biorthonormal, i.e. $\left\langle\phi_{p}^{A} \mid \phi_{q}^{B}\right\rangle=\delta_{p q} \cdot{ }^{59}$ When this is not the case, like in the work of Ferté et al., ${ }^{23}$ the more complicated Löwdin's rules have to be used to compute $\left\langle\phi_{p}^{A} \mid \phi_{q}^{B}\right\rangle$ and then $S_{\mu \nu}{ }^{69}$

However, it is possible to find a set of non-unitary orbital transformations which transform $\left\{\phi^{A}\right\},\left\{\phi^{B}\right\},\left\{C^{A}\right\},\left\{C^{B}\right\} \rightarrow\left\{\widetilde{\phi^{A}}\right\},\left\{\widetilde{\phi^{B}}\right\},\left\{\widetilde{C^{A}}\right\},\left\{\widetilde{C^{B}}\right\}$, such that the MO overlap $\left\langle\widetilde{\phi}_{p}^{A} \mid \widetilde{\phi}_{q}^{B}\right\rangle=$ $\delta_{p q}$, while the wave functions $\Psi_{A}$ and $\Psi_{B}$ remain the same. The procedure is described in detail in Ref. 65. Shortly, the biorthonormal orbital sets are obtained by decomposing the overlap matrix into block-triangular factors which: (a) transform the original orbital sets into a biorthonormal set and, (b) allow a sequence of single-orbital operations that transform the CI coefficients and that never produce any new SD. Using these biorthonormally-transformed orbital sets allow us to employ simpler equations for overlap matrix elements between the two different wave functions, as if one common orthonormal orbital set is used, that is,

$$
\left\langle\Psi_{A} \mid \Psi_{B}\right\rangle=\sum_{\mu} \widetilde{C}_{\mu}^{A} \widetilde{C}_{\mu}^{B}
$$

We have implemented the computation of the spectral strength of Eq. (4) according to Eq. (8) within the RASSI framework ${ }^{59,65}$ in a locally modified version of the OpenMolcas program package ${ }^{70}$ by using:

$$
\begin{aligned}
& \left|\Psi_{A}\right\rangle=\left|\Psi_{\mathrm{DCH}}^{N-2}\right\rangle \\
& \left|\Psi_{B}\right\rangle=a_{1 s}^{\alpha} a_{1 s}^{\beta}\left|\Psi_{\mathrm{GS}}^{N}\right\rangle
\end{aligned}
$$

where $\left|\Psi_{\mathrm{DCH}}^{N-2}\right\rangle$ is the double core-holed wave function and $a_{1 s}^{\alpha} a_{1 s}^{\beta}\left|\Psi_{\mathrm{GS}}^{N}\right\rangle$ is the ground-state wave function with the core spin-orbitals' occupations annihilated. As anticipated, we used RASSCF $^{38}$ to compute the ground and DCH states and subsequently improved them with MS-RASPT2 in order to include dynamic correlation effects. ${ }^{55-58}$ Thus, the two sets of MO orbitals $\left\{\phi^{A}\right\}$ and $\left\{\phi^{B}\right\}$ are biorthogonalized, followed by application of the annihilators to the GS wave function, which then simplifies the computation of the overlap, Eq. (8).

The approximation in Eq. (4) assumes that the kinetic energy dependence of the transition moment and, thus, the interaction between the two photoelectrons and the $(N-2)$ bound wave-function is negligible. The sudden approximation should be a good approximation for high kinetic energies of the photoelectrons, as it generally happens to be the case far above the double core ionization potential. 


\begin{tabular}{|c|c|c|c|c|}
\hline Molecule & $\begin{array}{c}\text { Abelian } \\
\text { point group }\end{array}$ & RAS1 & RAS2 & \# electrons/\# orbitals \\
\hline $\mathrm{H}_{2} \mathrm{O}$ & $C_{2 v}$ & $1 a_{1}\left(1 s_{\mathrm{O}}\right)$ & $2-7 a_{1}, 1-3 b_{1}, 1-3 b_{2}$ & $\operatorname{RAS}(10 / 13)$ \\
\hline $\mathrm{CO}\left(\mathrm{O} K^{-2}\right)$ & $C_{2 v}$ & $1 a_{1}\left(1 s_{\mathrm{O}}\right)$ & $4-7 a_{1}, 1-3 b_{1}, 1-3 b_{2}$ & $\operatorname{RAS}(10 / 11)$ \\
\hline $\mathrm{CO}\left(\mathrm{C} K^{-2}\right)$ & $C_{2 v}$ & $2 a_{1}\left(1 s_{\mathrm{C}}\right)$ & $4-7 a_{1}, 1-3 b_{1}, 1-3 b_{2}$ & $\operatorname{RAS}(10 / 11)$ \\
\hline $\mathrm{N}_{2}$ & $D_{2 h} \rightarrow C_{2 v}$ & $2 a_{1}\left(1 s_{\mathrm{N}}\right)$ & $3-8 a_{1}, 1-2 b_{1}, 1-2 b_{2}$ & $\operatorname{RAS}(12 / 11)$ \\
\hline $\mathrm{C}_{2} \mathrm{H}_{2}$ & $D_{2 h} \rightarrow C_{2 v}$ & $2 a_{1}\left(1 s_{\mathrm{C}}\right)$ & $3-8 a_{1}, 1-2 b_{1}, 1-2 b_{2}$ & $\operatorname{RAS}(12 / 11)$ \\
\hline $\mathrm{C}_{2} \mathrm{H}_{4}$ & $D_{2 h} \rightarrow C_{2 v}$ & $2 a_{1}\left(1 s_{\mathrm{C}}\right)$ & $3-7 a_{1}, 1-2 b_{1}, 1-4 b_{2}$ & $\operatorname{RAS}(14 / 12)$ \\
\hline $\mathrm{C}_{2} \mathrm{H}_{6}$ & $C_{2 h} \rightarrow C_{s}$ & $2 a^{\prime}\left(1 s_{\mathrm{C}}\right)$ & $3-10 a^{\prime}, 1-3 a^{\prime \prime}$ & $\operatorname{RAS}(16 / 12)$ \\
\hline
\end{tabular}

TABLE I: Active spaces used in the RASSCF/RASPT2 calculations.

We tested our approach by computing the ssDCH spectra of $\mathrm{CO}, \mathrm{H}_{2} \mathrm{O}, \mathrm{N}_{2}$ and of the $\mathrm{C}_{2} \mathrm{H}_{2 n}(n=1-3)$ series, and comparing our results with available experimental data. ${ }^{15,17,23}$ An imaginary level shift of 0.45 hartree was applied to avoid intruder state singularities in the RASPT2 calculations. Within the framework of the state interaction approximation, where a basis of biorthogonal orbital set is used to compute the matrix elements, we were able to optimize the ground state and DCH wave functions in separate calculations, securing a unbiased description of the relaxation and correlation effects. The correlation-consistent augmented triple zeta basis (aug-cc-pVTZ) set of Dunning and coworkers ${ }^{71,72}$ was employed throughout. Additional core-polarization functions (aug-cc-pCVTZ) were used for oxygen and nitrogen, when they are the relevant core edges. No sensible changes were noticed at the carbon edge of CO when using the aug-cc-pCVTZ basis set, beside an overall red shift (see Figure S2). The aug-cc-pVTZ basis was therefore employed in all subsequent calculations at the carbon edge. Douglas-Kroll-Hess scalar relativistic effects ${ }^{73}$ up to the second order were incorporated in all our calculations.

Since the selection of orbitals to form the RASSCF active space is system dependent, it is often necessary to test more than one active space until the desired result is reached. In Table I we report the active spaces of each molecule used in this work. The RAS approach can be used to avoid the variational collapse of the wave-function to a low-lying state. The RAS1 space contains the core-shell orbital where double hole condition is enforced by means of a core-valence separation technique implemented in OpenMolcas, ${ }^{47}$ while RAS2 is used 
for complete electron distribution. RAS3 was kept empty. For the highly symmetric $\mathrm{N}_{2}$ and $\mathrm{C}_{2} \mathrm{H}_{2 n}(n=1-3)$ molecules, where the core orbitals are delocalized, we apply a Cholesky localization procedure ${ }^{62}$ to localize the core orbitals. In the case of equivalent centers, the localization of core orbitals when aiming at the relevant states of a ssDCH spectrum had been suggested by Cederbaum et al. ${ }^{35}$ as an efficient procedure to address such states. The ssDCH states could alternatively be obtained without localizing the core by including both core orbitals in the RAS1 space. This, however, will require us to converge the manifold of two-site DCH states until the first ssDCH state is reached, resulting in unnecessary computational effort. Note that localizing core orbitals with the Cholesky procedure requires that the (core) orbitals to be localized belong to the same irreducible representation, otherwise they will maintain their delocalized nature. In full Abelian $D_{2 h}$ point group symmetry, the $1 s$ orbitals of $\mathrm{N}_{2}, \mathrm{C}_{2} \mathrm{H}_{2}$ and $\mathrm{C}_{2} \mathrm{H}_{4}$ belong to the $a_{g}$ and $b_{1 u}$ irreducible representations, which prevents straightforward application of the procedure. However, by reducing point group symmetry from $D_{2 h}$ to $C_{2 v}$, the $1 s$ delocalized orbitals now belong to the same $a_{1}$ irreducible representation, allowing us to use the Cholesky localization procedure. In $\mathrm{C}_{2} \mathrm{H}_{6}$, the two $\mathrm{C}$ $1 s$ delocalized orbitals belong to the $a_{1}$ and $b_{1}$ irreducible representations of the $C_{2 h}$ point group, but can be brought into the same $a^{\prime}$ irreducible representation by reducing the point group symmetry to $C_{s}$.

The DCH states were computed by state averaging over 15 final singlet states for $\mathrm{H}_{2} \mathrm{O}$, 30 singlet states for $\mathrm{CO}, 30$ singlet states $\mathrm{N}_{2}$, and 30 singlet states for each member of the $\mathrm{C}_{2} \mathrm{H}_{2 n}$ series.

All calculations were carried out at the experimental ground-state equilibrium geometries from the NIST Chemistry webBook database. ${ }^{74}$ To ease the comparison with the experimental results, the sticks spectra are convoluted with a Gaussian broadening of half-width-athalf-maximum (HWHM) between 0.5 and $1.5 \mathrm{eV}$, depending on the experimental spectrum we compare with. Additionally, the theoretical spectra were shifted on the energy scale by a value $\Delta$ indicated in the figures, in order to match the experimental position of the first peak. The experimental data points reproduced in our figures were digitized from the original references using WebPlotDigitizer. ${ }^{75}$ To avoid repetition, here we present and discuss only RASPT2 results. For RASSCF results, we refer the reader to the Supplemental Material.

In Figure 1 and Table II, we present the theoretical results for the $K^{-2}$ spectra of $\mathrm{H}_{2} \mathrm{O}$, 
$\mathrm{N}_{2}$, and CO. The experimental points (in red) were digitized from Ref. $15\left(\mathrm{H}_{2} \mathrm{O}\right)$, Ref. 17 $\left(\mathrm{N}_{2}\right)$, and Refs. 17, $23(\mathrm{CO})$. The ground-state HF occupied molecular orbitals are indicated as: $\left(\mathrm{H}_{2} \mathrm{O}\right) 1 s_{\mathrm{O}}^{2}, 2 a_{1}^{2}, 1 b_{2}^{2}, 3 a_{1}^{2}, 1 b_{1}^{2} ;\left(\mathrm{N}_{2}\right) 1 s_{\mathrm{N}}^{2}, 1 s_{\mathrm{N}}^{2}, 1 \sigma_{2 s}^{2}, 2 \sigma^{2}, 3 \sigma^{2}, 1 \pi^{4}$ and $(\mathrm{CO}) 1 s_{\mathrm{O}}^{2}, 1 s_{\mathrm{C}}^{2}$, $1 \sigma_{2 s}^{2}, 2 \sigma^{2}, 1 \pi^{4}, 3 \sigma^{2}$. The active space of CO was chosen as in Ref. 23.

\begin{tabular}{|c|c|c|c|c|}
\hline Molecule & State Label & $\mathrm{BE}(\mathrm{eV})$ & BE Exp. $(\mathrm{eV})$ & $\left|T_{i \rightarrow f}\right|^{2}$ \\
\hline & $\mathrm{A}$ & 1173.22 & $1171.0 \pm 1^{13,15}$ & 0.2411 \\
\hline \multirow[t]{4}{*}{$\mathrm{H}_{2} \mathrm{O}$} & B & 1198.53 & - & 0.0138 \\
\hline & $\mathrm{C}$ & 1203.18 & - & 0.0299 \\
\hline & $\mathrm{D}$ & 1206.70 & - & 0.0268 \\
\hline & $\mathrm{E}$ & 1208.74 & - & 0.0613 \\
\hline \multirow[t]{4}{*}{$\mathrm{N}_{2}$} & $\mathrm{~A}$ & 902.55 & $(902.5 \pm 1)^{17}$ & 0.2509 \\
\hline & $\mathrm{B}$ & 918.94 & $(919.0 \pm 1)^{17}$ & 0.0951 \\
\hline & $\mathrm{C}$ & 929.11 & - & 0.0169 \\
\hline & $\mathrm{D}$ & 934.27 & - & 0.0508 \\
\hline \multirow[t]{7}{*}{$\mathrm{CO}\left(K^{-2}\right)$} & A & 1177.20 & $(1178.0 \pm 0.8)^{23}$ & 0.2319 \\
\hline & B & 1192.69 & - & 0.0094 \\
\hline & $\mathrm{C}$ & 1196.56 & - & 0.0150 \\
\hline & $\mathrm{D}$ & 1199.81 & $(1200.6 \pm 0.8)^{23}$ & 0.0366 \\
\hline & $\mathrm{E}$ & 1200.29 & $(1200.6 \pm 0.8)^{23}$ & 0.0835 \\
\hline & $\mathrm{F}$ & 1214.31 & - & 0.0206 \\
\hline & G & 1216.57 & - & 0.0365 \\
\hline \multirow[t]{5}{*}{$\mathrm{C}\left(K^{-2}\right) \mathrm{O}$} & $\mathrm{A}$ & 665.76 & $(666.7 \pm 1)^{17}$ & 0.3123 \\
\hline & B & 682.32 & $(682.2 \pm 1)^{17}$ & 0.0676 \\
\hline & $\mathrm{C}$ & 693.56 & - & 0.0318 \\
\hline & $\mathrm{D}$ & 696.25 & - & 0.0239 \\
\hline & $\mathrm{E}$ & 698.32 & - & 0.0235 \\
\hline
\end{tabular}

TABLE II: RASPT2 $K^{-2}$ binding energies (BE) and spectral strengths of the main peaks of $\mathrm{CO}(\mathrm{C}$ and $\mathrm{O}$ edges), $\mathrm{H}_{2} \mathrm{O}$ and $\mathrm{N}_{2}$.

The computed $K^{-2}$ spectrum of $\mathrm{H}_{2} \mathrm{O}$, shown in Figure 1a, exhibits five main peaks labeled 
A-E, with binding energies and spectral strengths listed in Table II. Peak A corresponds to a double photoionization from the oxygen's $1 s$ orbital, where the weight of the major configuration in the $\mathrm{CI}$ expansion is $0.96\left(1 s_{\mathrm{O}}^{-2}\right)$. The spectral strength computed with the approximation given by Eq. (4) is a monopolar transition between the initial ground state and a final double core state. As a consequence, the more similar the final state is to the ground state configuration, the more intense the transition will be. This also means that almost pure double-core photoionized final states will have more overlap with the ground state than multi-configurational DCH shake-up states. Therefore, we in general expect the pure double-core photoionized state to be the most intense peak in the DCH spectrum. The main CI configurations and weights of the shake-up satellite peaks B, C and D are, respectively: $0.96\left(1 s_{\mathrm{O}}^{-2} 3 a_{1}^{-1} 4 a_{1}^{1}\right), 0.87\left(1 s_{\mathrm{O}}^{-2} 1 b_{2}^{-1} 2 b_{2}^{1}\right)$ and $0.79\left(1 s_{\mathrm{O}}^{-2} 1 b_{1}^{-1} 2 b_{1}^{1}\right)$. The multi-configuration character of peak $\mathrm{E}$ is more pronounced than for its predecessors, being $0.62\left(1 s_{\mathrm{O}}^{-2} 3 a_{1}^{-1} 7 a_{1}^{1}\right)+$ $0.16\left(1 s_{\mathrm{O}}^{-2} 3 a_{1}^{-1} 6 a_{1}^{1}\right)+0.12\left(1 s_{\mathrm{O}}^{-2} 1 b_{1}^{-1} 2 b_{1}^{1}\right)$, where the orbitals $6-7 a_{1}$ are atomic-like orbitals of $s$ type. Notice that we are only showing configurations with weights higher than $0.1(10 \%)$ in the CI expansion, which means that a large number of CI configurations, important to recover relaxation/correlation effects, are not listed here. Unfortunately, the available experimental data ${ }^{15}$ only cover a limited energy range, from 1160 to $1200 \mathrm{eV}$, where it is impossible to observe all the computed shake-up satellites. However, one can notice an increase in the experimental intensity after $1190 \mathrm{eV}$, where the first satellite, labeled B, appears.

The RASSCF $K^{-2}$ spectrum of $\mathrm{H}_{2} \mathrm{O}$ is shown in Figure S1a. It exhibits the same peaks present in the RASPT2 spectrum, but slightly less intense from peaks B to D. Overall, we observe a good agreement between the $K^{-2}$ spectra of $\mathrm{H}_{2} \mathrm{O}$ computed with RASSCF and those obtained with RASPT2. The RASSCF energies and intensities can be consulted in Table S1. Moreover, the shifts relative to the reference value ${ }^{13}$ go from $\Delta=-2.2 \mathrm{eV}$ with RASPT2 to $\Delta=-3.4 \mathrm{eV}$ with RASSCF.

We note that the experimental binding energy (BE) reported in Ref. 15 for peak A was calibrated using the CASSCF/cc-pCVTZ result from Ref. 13, obtained with 10 active electrons and 6 orbitals, resulting in a binding energy of $1170.85 \mathrm{eV}$. Another BE obtained at the $\Delta \mathrm{CI}$ /aug-cc-pCV5Z level including relativistic correction has been reported as 1171.98 $\mathrm{eV} .{ }^{15}$ The BE reported in Ref. 13 is noticeably distant from the RASSCF and RASPT2 BEs reported here, computed with a larger active space/basis set. Our shifts relative to the $\Delta \mathrm{CI}$ value go from $\Delta=-1.2 \mathrm{eV}$ with RASPT2 to $\Delta=-2.4 \mathrm{eV}$ with RASSCF. 
(a) $\mathrm{H}_{2} \mathrm{O}$
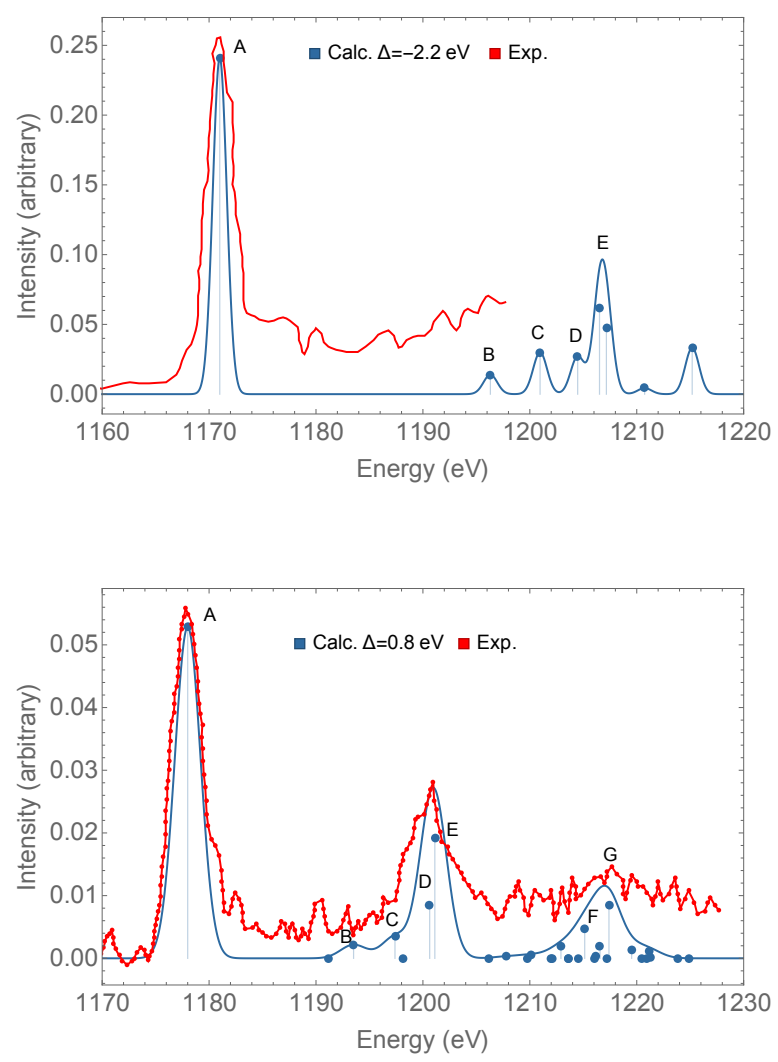

(c) $\mathrm{CO}(\mathrm{O} \mathrm{ssDCH})$ (b) $\mathrm{N}_{2}$
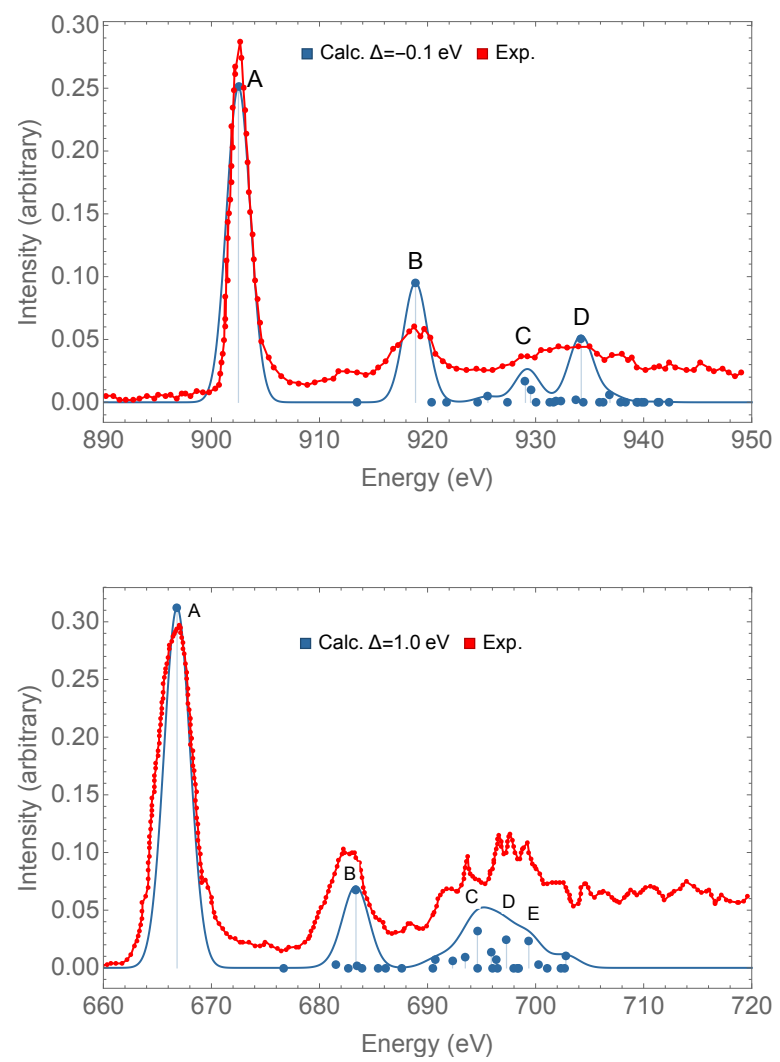

(d) $\mathrm{CO}(\mathrm{C} \mathrm{ssDCH})$

FIG. 1: RASPT2 $K^{-2}$ spectra of $\mathrm{H}_{2} \mathrm{O}, \mathrm{N}_{2}$, and CO. The experimental points were extracted from Ref. 15 for $\mathrm{H}_{2} \mathrm{O}$, Ref. 17 for $\mathrm{N}_{2}$, and Refs. 17 and 23 for CO. The aug-cc-pCVTZ basis was used for the $\mathrm{O}$ and $\mathrm{N}$ atoms when they are the relevant edges, and aug-cc-pVTZ on the remaining atoms. The theoretical spectra were shifted by $\Delta$, whose value is given in the legends, to match the position of the first experimental peak. The stick spectra were broadened with Gaussian functions using a HWHM of $0.7 \mathrm{eV}$ for $\mathrm{H}_{2} \mathrm{O}$ and and of $1.1 \mathrm{eV}$ for $\mathrm{N}_{2}$ and $\mathrm{CO}$.

The $K^{-2}$ spectrum of $\mathrm{N}_{2}$ is shown in Figure $1 \mathrm{~b}$ together with experimental data. ${ }^{17}$ Overall, the agreement between theory and experiment is good. The four main peaks contributing to the spectrum are labeled A-D and their binding energies and spectral strengths are listed in Table II. Peak A has a major contribution from the double photoionization from one of the $\mathrm{N} 1 s$ orbital. The weight of the $1 s_{\mathrm{N}}^{-2}$ configuration in the CI expansion is 0.81 . Peak B corresponds to the $1 s_{\mathrm{N}}^{-2}$ hole and a $\pi \rightarrow \pi^{*}$ valence excitation, with main CI configuration and weight $0.66\left(1 s_{\mathrm{N}}^{-2} 1 \pi^{-1} \pi^{* 1}\right)$, and dozens of other configurations with weights less than 
0.1. The binding energy of this peak is in good agreement with experiment, although its intensity is higher. This might be due to energy dissipation or nuclear relaxation effects not taken into account in our calculations. Another possible reason for the higher intensity on peak B could be the approximate treatment to the bound-to-continuum matrix element given by Eq. 4. Peaks $\mathrm{C}$ and $\mathrm{D}$ belong to the high energy region of the spectrum, where a broad and flat band is observed at around $930 \mathrm{eV}$. Both states $\mathrm{C}$ and $\mathrm{D}$ present strong multi-configuration character, with two electrons removed from the $\mathrm{N} 1 s$ orbital followed by a $3 \sigma \rightarrow \sigma^{*}(\mathrm{C})$ or $2 \sigma \rightarrow \sigma^{*}(\mathrm{D})$ valence excitations.

Comparing the $K^{-2}$ spectra of $\mathrm{N}_{2}$ obtained with the RASSCF and RASPT2 approaches (Figure S1b and Figure 1b, respectively), we notice that the only sensitive improvement brought by the RASPT2 method is on peak B, which is slightly more accurate with RASPT2. The $\Delta$ values from the two methods are also very similar, which indicates that the RASSCF results are as good as the RASPT2 ones for the $\mathrm{N}_{2}$ molecule.

The $K^{-2}$ spectra of $\mathrm{CO}$ were computed both at the oxygen and carbon edges. The results are presented in Figures $1 \mathrm{c}$ and $1 \mathrm{~d}$ alongside with experimental data. ${ }^{17,23}$ In both cases, the agreement between the experimental results and the theoretical spectra is very good, if not excellent. The experimental profile of the $\mathrm{O} K^{-2}$ spectrum shows three prominent features, the first being a strong peak centered at $1178 \mathrm{eV}$, followed by a broad peak at $1200 \mathrm{eV}$ and a very broad and weak band around $1220 \mathrm{eV}$. The spectrum shown in Figure 1c displays seven main contributing 'sticks' that we labeled A-G. The corresponding binding energies and spectral strengths can be consulted in Table II. Peak A corresponds, as usual, to the double photoionization from the $\mathrm{O} 1 s$ orbital with weight of the main configuration in the $\mathrm{CI}$ expansion $0.90\left(1 \mathrm{~s}_{\mathrm{O}}^{-2}\right)$. In the second region of the spectrum, centered at $1200 \mathrm{eV}$, we identify four peaks, B-E, where $\mathrm{E}$ is the brighter. The shake-up satellite state B is associated with the main CI configurations $0.82\left(1 s_{\mathrm{O}}^{-2} 3 \sigma^{-2} 2 \pi^{* 2}\right)$, whereas peak $\mathrm{C}$ is associated with the main configurations $0.61\left(1 s_{\mathrm{O}}^{-2} 3 \sigma^{-1} 4 \sigma^{* 1}\right)+0.22\left(1 s_{\mathrm{O}}^{-2} 1 \pi^{-1} 2 \pi^{* 1}\right)$. The shake-up satellites $\mathrm{D}$ and $\mathrm{E}$ are associated with the main CI configurations $0.38\left(1 s_{\mathrm{O}}^{-2} 3 \sigma^{-1} 4 \sigma^{* 1}\right)+0.10\left(1 s_{\mathrm{O}}^{-2} 3 \sigma^{-1} 5 \sigma^{* 1}\right)$ $+0.38\left(1 s_{\mathrm{O}}^{-2} 1 \pi^{-1} 2 \pi^{* 1}\right)$ for peak $\mathrm{D}$, and the main CI configurations $0.44\left(1 s_{\mathrm{O}}^{-2} 3 \sigma^{-1} 4 \sigma^{* 1}\right)+$ $0.13\left(1 \mathrm{~s}_{\mathrm{O}}^{-2} 3 \sigma^{-1} 5 \sigma^{* 1}\right)+0.22\left(1 s_{\mathrm{O}}^{-2} 1 \pi^{-1} 2 \pi^{* 1}\right)$ for peak E. Overall, the relative intensities between peaks A and D-E are in excellent agreement with those from the experimental spectrum, which we attribute to the optimal description of the non-orthogonal states given by the RASPT2/state-interaction approximation. ${ }^{65}$ Our theoretical assignments and binding 
energies for the oxygen $K^{-2}$ spectrum of $\mathrm{CO}$ agree well with the results obtained by Ferté et al. using the NOTA + CIPSI approach. ${ }^{23}$ The last region around $1215 \mathrm{eV}$ is dominated by the satellite states $\mathrm{F}$ and $\mathrm{G}$, with main configurations $0.70\left(1 s_{\mathrm{O}}^{-2} 1 \pi^{-1} 3 \pi^{* 1}\right)$ for $\mathrm{F}$, and $0.43\left(1 s_{\mathrm{O}}^{-2} 2 \sigma^{-1} 4 \sigma^{* 1}\right)+0.20\left(1 s_{\mathrm{O}}^{-2} 3 \sigma^{-1} 4 \sigma^{* 1} 1 \pi^{-1} 3 \pi^{* 1}\right)$ for $\mathrm{G}$.

The $\mathrm{C} K^{-2}$ spectrum of $\mathrm{CO}$ is shown in Figure 1d. As the experiment shows,${ }^{17}$ the $\mathrm{C}$ $K^{-2}$ spectrum is also characterized by three main regions. The first important feature is an intense peak centered at $665 \mathrm{eV}$, followed by a weaker peak centered at $682 \mathrm{eV}$, and a broad region above $690 \mathrm{eV}$ with many distinct unsolved structures. One can notice that the experimental spectrum at the carbon edge is less noisy than the one at the oxygen edge, which makes it easier to identity the shake-up satellites structures in the spectrum. The first feature, labeled A in Figure 1d and Table II, is again the pure double photoionization from the $\mathrm{C} 1 s$ orbital, with weight of the major configuration in the $\mathrm{CI}$ expansion being $0.84\left(1 \mathrm{~s}_{\mathrm{C}}^{-2}\right)$. Peak $\mathrm{B}$ is characterized by the main configurations $0.21\left(1 s_{\mathrm{C}}^{-2} 3 \sigma^{-1} 4 \sigma^{* 1}\right)+0.50\left(1 \mathrm{~s}_{\mathrm{C}}^{-2} 1 \pi^{-1} 2 \pi^{* 1}\right)$ and a large number of secondary configurations with small weights. The upper region of the spectrum, after $690 \mathrm{eV}$, presents a large number of shake-up satellites, of which we selected three with relevant intensities and labeled them C-E. Peaks C-E present strong multi-excitation character where a large number of singles and doubles valence $1 s_{\mathrm{C}}^{0} \sigma \rightarrow \sigma^{*}$ and $1 s_{\mathrm{C}}^{0} \pi \rightarrow \pi^{*}$ excitations can be identified. Nonetheless, peaks C-E demonstrate good agreement with the aggregate experimental structures present in this region $\mathrm{C} K^{-2}$ spectrum of $\mathrm{CO}$.

As also observed for the $\mathrm{N}_{2}$ molecule, the RASSCF and RASPT2 $K^{-2}$ spectra of CO at both the carbon and the oxygen edges are in very good agreement with each other. The carbon $K^{-2}$ spectrum computed with RASPT2 (Figure 1d) shows slightly better agreement with experiment for peak B than the RASSCF result (Figure S1d, which anyway is also in good agreement with experiment. Interestingly, the absolute values of $\Delta$ obtained with RASSCF (Figures S1c and S1d) are even slightly smaller than the ones obtained with RASPT2 (Figures 1c and 1d). The difference is in any case too small to conclude that one is more accurate than the other.

Having demonstrated the accuracy of our method for the $K^{-2}$ spectra of $\mathrm{H}_{2} \mathrm{O}, \mathrm{N}_{2}$ and $\mathrm{CO}$, we now characterize the spectra of ethyne, ethene and ethane. In Figure 2 we show the computed $K^{-2}$ spectra plotted alongside with experiment. ${ }^{17}$ The binding energies and spectral strengths are listed in Table III. The occupied orbitals in the HF ground state are: 


\begin{tabular}{lcccc}
\hline Molecule & State Label BE $(\mathrm{eV})$ & BE Exp. (eV) $\left|T_{i \rightarrow f}\right|^{2}$ \\
\hline $\mathrm{C}_{2} \mathrm{H}_{2}$ & A & 651.95 & $652.5 \pm 0.5$ & 0.2385 \\
& B & 665.43 & $666.5 \pm 0.5$ & 0.0905 \\
& C & 677.25 & - & 0.0310 \\
& D & 678.27 & - & 0.0423 \\
\hline $\mathrm{C}_{2} \mathrm{H}_{4}$ & A & 649.91 & $650.4 \pm 0.5$ & 0.1971 \\
& B & 660.10 & $660.5 \pm 0.5$ & 0.0925 \\
& C & 673.56 & - & 0.0416 \\
& D & 677.97 & - & 0.0146 \\
\hline $\mathrm{C}_{2} \mathrm{H}_{6}$ & A & 649.55 & $650.5 \pm 0.5$ & 0.2406 \\
& B & 666.91 & - & 0.0098 \\
& C & 671.58 & - & 0.0129 \\
\hline$\Delta E_{\mathrm{C}_{2} \mathrm{H}_{4} / \mathrm{C}_{2} \mathrm{H}_{2}}$ & & -2.04 & $-2.1 \pm 0.2$ & \\
$\Delta E_{\mathrm{C}_{2} \mathrm{H}_{6} / \mathrm{C}_{2} \mathrm{H}_{2}}$ & & -2.40 & $-1.9 \pm 0.2$ & \\
\hline
\end{tabular}

TABLE III: $K^{-2}$ binding energies and intensity of the main peaks of $\mathrm{C}_{2} \mathrm{H}_{2 n}(n=1-3)$ computed at the RASPT2 level. The experimental results were obtained from Ref. 17.

$1 s_{\mathrm{C}}^{2}, 1 s_{\mathrm{C}}^{2}, 1 \sigma_{2 s}^{2}, 2 \sigma_{\mathrm{C}-\mathrm{C}}^{2}, 3 \sigma_{\mathrm{C}-\mathrm{C}}^{2}, 1 \pi^{4}$ for $\mathrm{C}_{2} \mathrm{H}_{2} ; 1 s_{\mathrm{C}}^{2}, 1 s_{\mathrm{C}}^{2}, 1 \sigma_{2 s}^{2}, 2 \sigma_{\mathrm{C}-\mathrm{C}}^{2}, 3 \sigma_{s p^{2}}^{2}, 4 \sigma_{\mathrm{C}-\mathrm{C}}^{2}, 5 \sigma_{s p^{2}}^{2}, 1 \pi^{2}$ for $\mathrm{C}_{2} \mathrm{H}_{4}$ and $1 s_{\mathrm{C}}^{2}, 1 s_{\mathrm{C}}^{2}, 1 \sigma_{2 s}^{2}, 2 \sigma_{\mathrm{C}-\mathrm{C}}^{2}, 3 \sigma_{s p^{3}}^{2}, 4 \sigma_{s p^{3}}^{2}, 5 \sigma_{\mathrm{C}-\mathrm{C}}^{2}, 6 \sigma_{\mathrm{CH}}^{2}, 7 \sigma_{\mathrm{CH}}^{2}$ for $\mathrm{C}_{2} \mathrm{H}_{6}$.

The experimental spectrum of $\mathrm{C}_{2} \mathrm{H}_{2}{ }^{17}$ shows three well separated features: a bright peak centered at $652 \mathrm{eV}$, labeled peak $\mathrm{A}$ in Figure 2a, followed by a second peak centered at $666 \mathrm{eV}$, labeled B, and a broad and relatively weak structure starting at around $675 \mathrm{eV}$. We obtained two peaks contributing to this last region that we labeled $\mathrm{C}$ and $\mathrm{D}$. The binding energies and intensities can be consulted in Table III. The relative energies and intensities between peaks A and B look very precise, while our computed peak D is slightly more intense than the corresponding experimental one. Overall, we find good agreement between the experiment and the computed results throughout the spectrum. Peak $\mathrm{A}$ is a pure double photoionization from the $\mathrm{C} 1 s$ orbital. The weight of the major configuration in the CI expansion is $0.86\left(1 s_{\mathrm{C}}^{-2}\right)$. The shake-up peak B is associated with the main CI configuration and weight $0.74\left(1 s_{\mathrm{C}}^{-2} 1 \pi^{-1} 2 \pi^{* 1}\right)$ and a number of other configurations with small weights. Peak $\mathrm{C}$ is associated with the main configuration $0.47\left(1 s_{\mathrm{C}}^{-2} 3 \sigma^{-1} 4 \sigma^{* 1}\right)$, while 
(a) $\mathrm{C}_{2} \mathrm{H}_{2}$
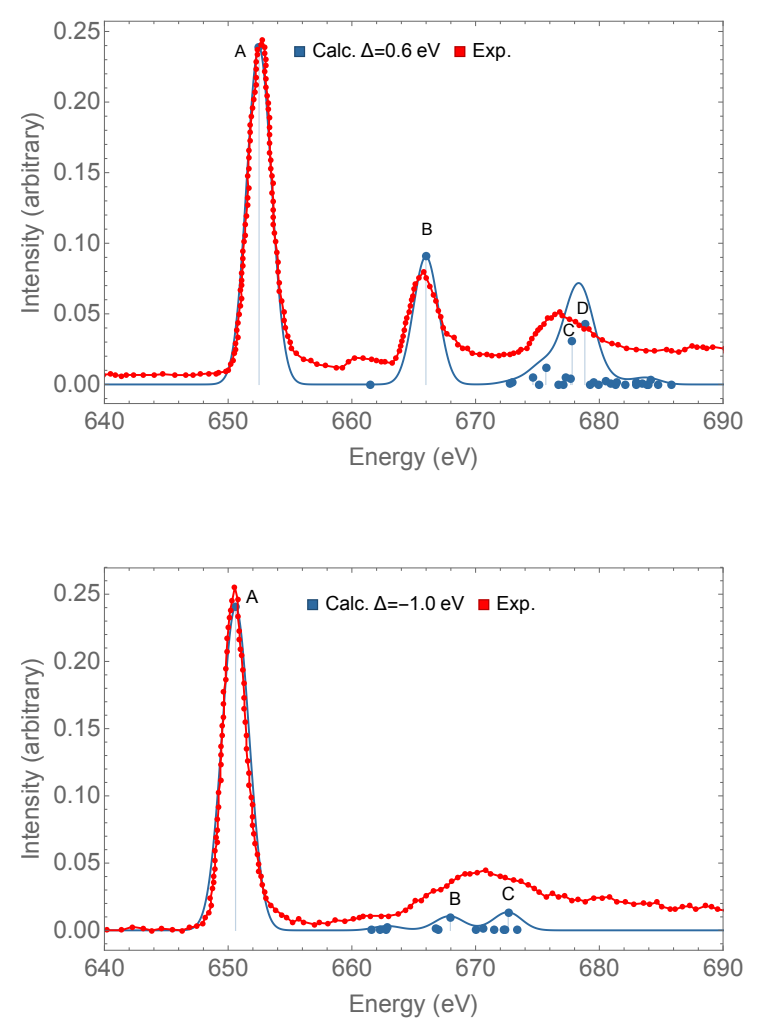

(c) $\mathrm{C}_{2} \mathrm{H}_{6}$ (b) $\mathrm{C}_{2} \mathrm{H}_{4}$
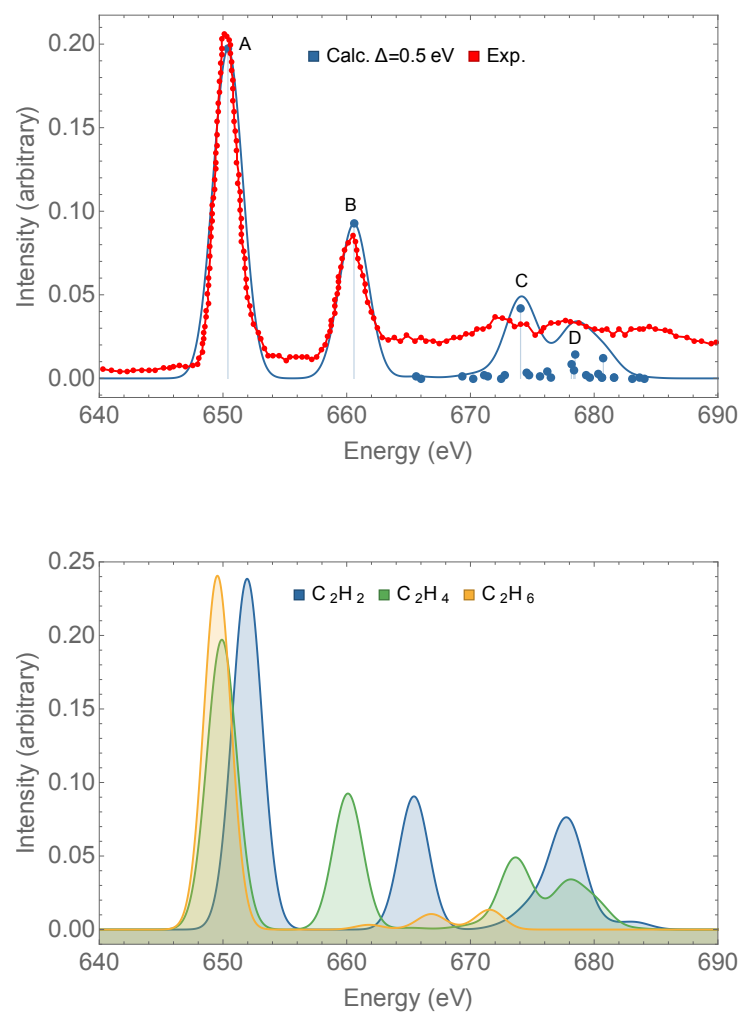

(d) $\mathrm{C}_{2} \mathrm{H}_{2 n}(n=1-3)$

FIG. 2: RASPT2 $K^{-2}$ spectra of $\mathrm{C}_{2} \mathrm{H}_{2 n}(n=1-3)$. The experimental points were re-digitized from Ref. 17 .

The aug-cc-pVTZ basis set was used on all atoms. The rigid shifts $\Delta$ used to align the computed spectra with the position of the first experimental peak are given in legend. The stick spectra were broadened using Gaussian functions with HWHM $=1.1 \mathrm{eV}$.

peak $\mathrm{D}$ is mainly associated with the multi-excited configurations $0.57\left(1 s_{\mathrm{C}}^{-2} 1 \pi^{-2} 2 \pi^{* 2}\right)$ and a large number of secondary configurations with small weights. Fair agreement is observed between the RASSCF (Figure S3a and the RASPT2 (Figure 2a) results. The $\Delta$ values at the two levels of approximation are similar, with RASPT2 result evidently more accurate relative to the experiment, especially with respect to peak B. The RASSCF result is more than $2 \mathrm{eV}$ higher that the experimental energy for this peak.

The $K^{-2}$ spectrum of $\mathrm{C}_{2} \mathrm{H}_{4}$ is displayed in Figure 2b. The first peak, labeled A, has weight $0.94\left(1 s_{\mathrm{C}}^{-2}\right)$ in the CI expansion. It was experimentally observed ${ }^{17}$ that the first peak of the $\mathrm{C}_{2} \mathrm{H}_{4} K^{-2}$ spectrum is red-shifted with respect to peak $\mathrm{A}$ in the $K^{-2}$ spectrum of 
$\mathrm{C}_{2} \mathrm{H}_{2}$. The experimental red-shift $\left(\Delta E_{\mathrm{C}_{2} \mathrm{H}_{4} / \mathrm{C}_{2} \mathrm{H}_{2}}\right)$ is $-2.1 \pm 0.2 \mathrm{eV}$, while the computed redshift is $-2.04 \mathrm{eV}$ (cf. Table III). Moreover, the relative binding energies and intensities between peaks A and B accurately reproduce the experimental profile. Peak B is a shake-up satellite associated with the main CI configurations $0.84\left(1 s_{\mathrm{C}}^{-2} 1 \pi^{-1} 2 \pi^{* 1}\right)$ and a large number of secondary configurations with small weights. The weak peaks $\mathrm{C}$ and $\mathrm{D}$ belong to the region above $670 \mathrm{eV}$ with various small and scattered structures, where it is very difficult to characterize individual contributions from the experiment. These peaks are associated with the main CI configurations: (peak C) $0.35\left(1 s_{\mathrm{C}}^{-2} 5 \sigma_{s p^{2}}^{-1} \sigma_{\mathrm{CH}}^{* 1}\right)+0.26\left(1 s_{\mathrm{C}}^{-2} 4 \sigma_{\mathrm{CC}}^{-1} \sigma_{\mathrm{CC}}^{* 1}\right)$ and (peak D) $0.60\left(1 s_{\mathrm{C}}^{-2} 1 \pi^{-2} 2 \pi^{* 2}\right)$. Excellent agreement is seen between the RASSCF (Figure S3b and the RASPT2 (Figure 2b) result for all the spectral features of the $\mathrm{C}_{2} \mathrm{H}_{4}$ molecule.

The $K^{-2}$ spectrum of $\mathrm{C}_{2} \mathrm{H}_{6}$ (Figure 2c) is a bit simpler than those of the unsaturated species, featuring only one single, strong, peak, labeled A, and a broad and weak region above $660 \mathrm{eV}$. Once again, the first peak is related to a pure double photoionized C $1 \mathrm{~s}$ state. The weak peaks labeled $\mathrm{B}$ and $\mathrm{C}$ are associated with the main CI configurations (B) $0.25\left(1 s_{\mathrm{C}}^{-2} 5 \sigma_{\mathrm{CC}}^{-1} \sigma_{\mathrm{CC}}^{* 1}\right)+0.16\left(1 s_{\mathrm{C}}^{-2} 5 \sigma_{\mathrm{CC}}^{-1} \sigma^{* 1}\right)$ and $(\mathrm{C}) 0.23\left(1 s_{\mathrm{C}}^{-2} 2 \sigma_{\mathrm{CC}}^{-1} \sigma_{\mathrm{CC}}^{* 1}\right)+0.18\left(1 s_{\mathrm{C}}^{-2} 2 \sigma_{\mathrm{CC}}^{-1} \sigma_{\mathrm{CH}}^{* 1}\right)$ $+0.12\left(1 s_{\mathrm{C}}^{-2} 4 \sigma_{s p^{3}}^{-1} \sigma_{\mathrm{CH}}^{* 1}\right)$. The computed red-shift on peak $\mathrm{A}\left(\Delta E_{\mathrm{C}_{2} \mathrm{H}_{6} / \mathrm{C}_{2} \mathrm{H}_{2}}\right)$ is $-2.40 \mathrm{eV}$, whereas the experimental value is $-1.9 \pm 0.2 \mathrm{eV}^{17}$. The RASSCF spectrum (Figure S3c) also shows good agreement with experiment. Remarkably, the $\Delta$ value obtained with RASSCF, $\Delta=0.1 \mathrm{eV}$, is significantly smaller than the corresponding RASPT2 value, $\Delta=1.0 \mathrm{eV}$.

In Figure $2 \mathrm{~d}$ we compare the results for the $\mathrm{C}_{2} \mathrm{H}_{2 n}(n=1-3) K^{-2}$ spectra jointly, where the shifts of peak A are clearly apparent. As a general remark, we were able to reproduce and characterize the main experimental features in the $K^{-2}$ spectra of $\mathrm{C}_{2} \mathrm{H}_{2 n}(n=1-3)$ with excellent accuracy.

To summarize, we have presented a highly accurate method to compute the ssDCH spectra of general molecular systems, i.e., ssDCH binding energies and transition amplitudes. It is based on the calculation of (non-orthogonal) multi-reference wave-functions, conveniently obtained using the RASSCF ${ }^{37-39}$ and MS-RASPT2 $2^{57,58}$ methods. The transition amplitudes are estimated in the sudden approximation limit $^{76}$ by means of a monopolar matrix element $\left|T_{i \rightarrow f}\right|^{2}$, which is very efficiently computed within the state-interaction ${ }^{59}$ framework using biorthonormalized molecular orbital sets. The core orbitals of molecules with equivalent atoms, such as $\mathrm{N}_{2}$ and $\mathrm{C}_{2} \mathrm{H}_{2 n}(n=1-3)$, were localized using the Cholesky localization, which has the advantage of preserving some degree of point group symmetry while localizing, 
which is advantageous from a computational point of view. We were capable of reproducing the main experimental features of the $K^{-2}$ spectra of $\mathrm{H}_{2} \mathrm{O}, \mathrm{N}_{2}$, CO and the $\mathrm{C}_{2} \mathrm{H}_{2 n}(n=1-3)$ molecules with excellent agreement with experimental results. Our results and interpretation of the DCH spectrum of $\mathrm{CO}\left(\mathrm{O} K^{-2}\right.$ edge) were compared with previous theoretical $\operatorname{assignments}^{23}$, whereas the experimental shake-up satellite states of $\mathrm{CO}\left(\mathrm{C} \mathrm{K}^{-2}\right.$ edge $), \mathrm{H}_{2} \mathrm{O}$ and of the $\mathrm{C}_{2} \mathrm{H}_{2 n}(n=1-3)$ hydrocarbons were fully characterized for the first time here. The method is also quite practical, as we averaged a relatively small number of DCH states that is, between 15 and 30 states.

In perspective, we believe that the flexibility of the RASSCF method will permit us to apply our protocol to more challenging cases, for instance DCH states of bi-metal transition metal complexes, ${ }^{40}$ or imaging nuclear trajectories necessary to compute nuclear dynamics effects of organic molecules. We are currently exploring whether one can use the same RASSCF/State-Interaction framework to obtain the pre-edge DCH spectra ${ }^{8}$, coupling the direct and conjugate Dyson orbitals with an accurate continuum wave-function obtained at the (TD)DFT level on a B-spline basis. ${ }^{24}$ A detailed report of the pre-edge DCH methodology will be presented elsewhere.

\section{SUPPLEMENTARY MATERIAL}

See Supplementary Material for RASSCF results for all molecules, and RASPT2/aug-ccpCVTZ results at the $\mathrm{C}$ edge of $\mathrm{CO}$.

\section{ACKNOWLEDGMENTS}

This work was carried out with support from the Independent Research Fund DenmarkNatural Sciences, DFF-RP2 Grant No. 7014-00258B, and from the European Union's Horizon 2020 Research and Innovation Programme under the Marie Skłodowska-Curie Individual Fellowship (Grant Agreement 101027796). The European Cooperation in Science and Technology, COST Action CA18222, AttoChem, is also acknowledged. 


\title{
AUTHOR DECLARATIONS
}

\author{
Conflicts of Interest
}

The authors have no conflicts to disclose.

\section{DATA AVAILABILITY}

The data that support the findings of this study are available within the article and its supplementary material.

\section{REFERENCES}

${ }^{1}$ N. Berrah, L. Fang, B. Murphy, T. Osipov, K. Ueda, E. Kukk, R. Feifel, P. van der Meulen, P. Salen, H. T. Schmidt, R. D. Thomas, M. Larsson, R. Richter, K. C. Prince, J. D. Bozek, C. Bostedt, S.-i. Wada, M. N. Piancastelli, M. Tashiro, and M. Ehara, "Double-core-hole spectroscopy for chemical analysis with an intense X-ray femtosecond laser," Proc. Natl. Acad. Sci. 108, 16912-16915 (2011).

${ }^{2}$ P. Salén, P. van der Meulen, H. T. Schmidt, R. D. Thomas, M. Larsson, R. Feifel, M. N. Piancastelli, L. Fang, B. Murphy, T. Osipov, N. Berrah, E. Kukk, K. Ueda, J. D. Bozek, C. Bostedt, S. Wada, R. Richter, V. Feyer, and K. C. Prince, "Experimental verification of the chemical sensitivity of two-site double core-hole states formed by an x-ray free-electron laser," Phys. Rev. Lett. 108, 153003 (2012).

${ }^{3}$ M. Larsson, P. Salén, P. van der Meulen, H. T. Schmidt, R. D. Thomas, R. Feifel, M. N. Piancastelli, L. Fang, B. F. Murphy, T. Osipov, N. Berrah, E. Kukk, K. Ueda, J. D. Bozek, C. Bostedt, S. Wada, R. Richter, V. Feyer, and K. C. Prince, "Double core-hole formation in small molecules at the LCLS free electron laser," J. Phys. B: At. Mol. Opt. Phys. 46, 164030 (2013).

${ }^{4}$ V. Zhaunerchyk, M. Mucke, P. Salén, P. vd Meulen, M. Kaminska, R. J. Squibb, L. J. Frasinski, M. Siano, J. H. D. Eland, P. Linusson, R. D. Thomas, M. Larsson, L. Foucar, J. Ullrich, K. Motomura, S. Mondal, K. Ueda, T. Osipov, L. Fang, B. F. Murphy, N. Berrah, C. Bostedt, J. D. Bozek, S. Schorb, M. Messerschmidt, J. M. Glownia, J. P. Cryan, R. N. Coffee, O. Takahashi, S. Wada, M. N. Piancastelli, R. Richter, K. C. Prince, 
and R. Feifel, "Using covariance mapping to investigate the dynamics of multi-photon ionization processes of ne atoms exposed to X-FEL pulses," J. Phys. B: At. Mol. Opt. Phys. 46, 164034 (2013).

${ }^{5}$ L. J. Frasinski, V. Zhaunerchyk, M. Mucke, R. J. Squibb, M. Siano, J. H. D. Eland, P. Linusson, P. v.d. Meulen, P. Salén, R. D. Thomas, M. Larsson, L. Foucar, J. Ullrich, K. Motomura, S. Mondal, K. Ueda, T. Osipov, L. Fang, B. F. Murphy, N. Berrah, C. Bostedt, J. D. Bozek, S. Schorb, M. Messerschmidt, J. M. Glownia, J. P. Cryan, R. N. Coffee, O. Takahashi, S. Wada, M. N. Piancastelli, R. Richter, K. C. Prince, and R. Feifel, "Dynamics of Hollow Atom Formation in Intense X-Ray Pulses Probed by Partial Covariance Mapping," Phys. Rev. Lett. 111, 073002 (2013).

${ }^{6}$ D. Koulentianos, A. E. A. Fouda, S. H. Southworth, J. D. Bozek, J. Küpper, R. Santra, N. V. Kryzhevoi, L. S. Cederbaum, C. Bostedt, M. Messerschmidt, N. Berrah, L. Fang, B. Murphy, T. Osipov, J. P. Cryan, J. Glownia, S. Ghimire, P. J. Ho, B. Krässig, D. Ray, Y. Li, E. P. Kanter, L. Young, and G. Doumy, "High intensity x-ray interaction with a model bio-molecule system: double-core-hole states and fragmentation of formamide," J. Phys. B: At. Mol. Opt. Phys. 53, 244005 (2020).

${ }^{7}$ D. Koulentianos, S. Carniato, R. Püttner, G. Goldsztejn, T. Marchenko, O. Travnikova, L. Journel, R. Guillemin, D. Céolin, M. L. M. Rocco, M. N. Piancastelli, R. Feifel, and M. Simon, "Double-core-hole states in $\mathrm{CH}_{3} \mathrm{CN}$ : Pre-edge structures and chemical-shift contributions," J. Chem. Phys. 149, 134313 (2018).

${ }^{8}$ T. Marchenko, S. Carniato, G. Goldsztejn, O. Travnikova, L. Journel, R. Guillemin, I. Ismail, D. Koulentianos, J. Martins, D. Céolin, R. Püttner, M. N. Piancastelli, and M. Simon, "Single and multiple excitations in double-core-hole states of free water molecules," J. Phys. B: At. Mol. Opt. Phys. 53, 224002 (2020).

${ }^{9}$ J. H. D. Eland, M. Tashiro, P. Linusson, M. Ehara, K. Ueda, and R. Feifel, "Double core hole creation and subsequent auger decay in $\mathrm{nh}_{3}$ and $\mathrm{ch}_{4}$ molecules," Phys. Rev. Lett. 105, 213005 (2010).

${ }^{10}$ P. Lablanquie, F. Penent, J. Palaudoux, L. Andric, P. Selles, S. Carniato, K. Bučar, M. Žitnik, M. Huttula, J. H. D. Eland, E. Shigemasa, K. Soejima, Y. Hikosaka, I. H. Suzuki, M. Nakano, and K. Ito, "Properties of Hollow Molecules Probed by Single-Photon Double Ionization," Phys. Rev. Lett. 106, 063003 (2011). 
${ }^{11}$ P. Lablanquie, T. P. Grozdanov, M. Žitnik, S. Carniato, P. Selles, L. Andric, J. Palaudoux, F. Penent, H. Iwayama, E. Shigemasa, Y. Hikosaka, K. Soejima, M. Nakano, I. H. Suzuki, and K. Ito, "Evidence of Single-Photon Two-Site Core Double Ionization of $\mathbf{C}_{2} \mathbf{H}_{2}$ Molecules," Phys. Rev. Lett. 107, 193004 (2011).

${ }^{12}$ P. Linusson, O. Takahashi, K. Ueda, J. H. D. Eland, and R. Feifel, "Structure sensitivity of double inner-shell holes in sulfur-containing molecules," Phys. Rev. A 83, 022506 (2011).

${ }^{13}$ M. Mucke, J. H. D. Eland, O. Takahashi, P. Linusson, D. Lebrun, K. Ueda, and R. Feifel, "Formation and decay of core-orbital vacancies in the water molecule," Chem. Phys. Lett. 558, 82-87 (2013).

${ }^{14}$ M. Nakano, P. Selles, P. Lablanquie, Y. Hikosaka, F. Penent, E. Shigemasa, K. Ito, and S. Carniato, "Near-Edge X-Ray Absorption Fine Structures Revealed in Core Ionization Photoelectron Spectroscopy," Phys. Rev. Lett. 111, 123001 (2013).

${ }^{15}$ S. Carniato, P. Selles, L. Andric, J. Palaudoux, F. Penent, M. Žitnik, K. Bučar, M. Nakano, Y. Hikosaka, K. Ito, and P. Lablanquie, "Single photon simultaneous K-shell ionization and K-shell excitation. I. Theoretical model applied to the interpretation of experimental results on $\mathrm{H}_{2} \mathrm{O}$," J. Chem. Phys. 142, 014307 (2015).

${ }^{16}$ S. Carniato, P. Selles, L. Andric, J. Palaudoux, F. Penent, M. Žitnik, K. Bučar, M. Nakano, Y. Hikosaka, K. Ito, and P. Lablanquie, "Single photon simultaneous K-shell ionization and K-shell excitation. II. Specificities of hollow nitrogen molecular ions," J. Chem. Phys. 142, 014308 (2015).

${ }^{17}$ M. Nakano, F. Penent, M. Tashiro, T. P. Grozdanov, M. Žitnik, S. Carniato, P. Selles, L. Andric, P. Lablanquie, J. Palaudoux, E. Shigemasa, H. Iwayama, Y. Hikosaka, K. Soejima, I. H. Suzuki, N. Kouchi, and K. Ito, "Single Photon $K^{-2}$ and $K^{-1} K^{-1}$ Double Core Ionization in $\mathbf{C}_{2} \mathbf{H}_{2 n}(n=1-3), \mathbf{C O}$, and $\mathbf{N}_{2}$ as a Potential New Tool for Chemical Analysis," Phys. Rev. Lett. 110, 163001 (2013).

${ }^{18} \mathrm{~A}$. Hitchcock and C. Brion, "Carbon K-shell excitation of $\mathrm{C}_{2} \mathrm{H}_{2}, \mathrm{C}_{2} \mathrm{H}_{4}, \mathrm{C}_{2} \mathrm{H}_{6}$ and $\mathrm{C}_{6} \mathrm{H}_{6}$ by $2.5 \mathrm{keV}$ electron impact," Journal of Electron Spectroscopy and Related Phenomena 10, 317-330 (1977).

${ }^{19}$ J. van Bokhoven and C. Lamberti, eds., X-Ray Absorption and X-ray Emission Spectroscopy; Theory and Applications (Wiley \& Sons, 2016).

${ }^{20}$ S. Mobilio, F. Boscherini, and C. Meneghini, eds., Synchrotron Radiation: Basics, Methods and Applications (Springer, 2014). 
${ }^{21}$ C. Milne, T. Penfold, and M. Chergui, "Recent experimental and theoretical developments in time-resolved X-ray spectroscopies," Coord. Chem. Rev. 277-278, 44-68 (2014).

${ }^{22}$ U. Bergmann, V. Yachandra, and J. Yano, eds., X-Ray Free Electron Lasers: Applications in Materials, Chemistry and Biology, Energy and Environment Series No. 18 (Royal Society of Chemistry, 2017).

${ }^{23}$ A. Ferté, J. Palaudoux, F. Penent, H. Iwayama, E. Shigemasa, Y. Hikosaka, K. Soejima, K. Ito, P. Lablanquie, R. Taïeb, and S. Carniato, "Advanced Computation Method for Double Core Hole Spectra: Insight into the Nature of Intense Shake-up Satellites," J. Phys. Chem. Lett. 11, 4359-4366 (2020).

${ }^{24}$ A. Ponzi, C. Angeli, R. Cimiraglia, S. Coriani, and P. Decleva, "Dynamical photoionization observables of the CS molecule: The role of electron correlation," J. Chem. Phys. 140, 204304 (2014).

${ }^{25}$ A. Ponzi, N. Quadri, C. Angeli, and P. Decleva, "Electron correlation effects in the photoionization of co and isoelectronic diatomic molecules," Phys. Chem. Chem. Phys. 21, 1937-1951 (2019).

${ }^{26}$ H. Ågren and H. J. A. Jensen, "An efficient method for the calculation of generalized overlap amplitudes for core photoelectron shake-up spectra," Chem. Phys. Lett. 137, 431436 (1987).

${ }^{27}$ J. Wenzel, A. Holzer, M. Wormit, and A. Dreuw, "Analysis and Comparison of CVS-ADC Approaches up to Third Order for the Calculation of Core-Excited States," J. Chem. Phys. 142, $214104(2015)$.

${ }^{28}$ M. Wormit, D. R. Rehn, P. H. Harbach, J. Wenzel, C. M. Krauter, E. Epifanovsky, and A. Dreuw, "Investigating excited electronic states using the algebraic diagrammatic construction (adc) approach of the polarisation propagator," Mol. Phys. 112, 774-784 (2014).

${ }^{29}$ A. Dreuw and M. Wormit, "The algebraic diagrammatic construction scheme for the polarization propagator for the calculation of excited states," WIREs Comput. Mol. Sci. 5, 82-95 (2015).

${ }^{30}$ J. F. Stanton and R. J. Bartlett, "The equation of motion coupled-cluster method. a systematic biorthogonal approach to molecular excitation energies, transition probabilities, and excited state properties," J. Chem. Phys. 98, 7029-7039 (1993).

${ }^{31}$ M. L. Vidal, X. Feng, E. Epifanovsky, A. Krylov, and S. Coriani, "A New and Efficient Equation-of-Motion Coupled-Cluster Framework for Core-Excited and Core-Ionized 
States," J. Chem. Theory Comput. 15, 3117-3133 (2019).

${ }^{32}$ B. N. C. Tenorio, T. Moitra, M. A. C. Nascimento, A. B. Rocha, and S. Coriani, "Molecular inner-shell photoabsorption/photoionization cross sections at core-valence-separated coupled cluster level: Theory and examples," J. Chem. Phys. 150, 224104 (2019).

${ }^{33}$ L. S. Cederbaum, "Many-body theory of multiple core holes," Phys. Rev. A 35, 622-631 (1987).

${ }^{34}$ L. S. Cederbaum, F. Tarantelli, A. Sgamellotti, and J. Schirmer, "Double vacancies in the core of benzene," J. Chem. Phys. 86, 2168-2175 (1987).

${ }^{35}$ L. S. Cederbaum, F. Tarantelli, A. Sgamellotti, and J. Schirmer, "On double vacancies in the core," J. Chem. Phys. 85, 6513-6523 (1986).

${ }^{36}$ H. Ågren and H. J. A. Jensen, "Relaxation and correlation contributions to molecular double core ionization energies," Chem. Phys. 172, 45-57 (1993).

${ }^{37}$ B. O. Roos, P. R. Taylor, and P. E. M. Siegbahn, "A complete active space SCF method (CASSCF) using a density matrix formulated super-CI approach," Chem. Phys. 48, 157$173(1980)$.

${ }^{38}$ J. Olsen, B. O. Roos, P. Jørgensen, and H. J. A. Jensen, "Determinant based configuration interaction algorithms for complete and restricted configuration interaction spaces," J. Chem. Phys. 89, 2185-2192 (1988).

${ }^{39}$ P. A. Malmqvist, A. Rendell, and B. O. Roos, "The restricted active space self-consistentfield method, implemented with a split graph unitary group approach," J. Phys. Chem. 94, 5477-5482 (1990).

${ }^{40}$ Y. Zhang, U. Bergmann, R. Schoenlein, M. Khalil, and N. Govind, "Double core hole valence-to-core x-ray emission spectroscopy: A theoretical exploration using timedependent density functional theory," J. Chem. Phys. 151, 144114 (2019).

${ }^{41}$ Y. Zhang, D. Healion, J. D. Biggs, and S. Mukamel, "Double-core excitations in formamide can be probed by x-ray double-quantum-coherence spectroscopy," J. Chem. Phys. 138, 144301 (2013).

${ }^{42}$ O. Takahashi, M. Tashiro, M. Ehara, K. Yamasaki, and K. Ueda, "Theoretical spectroscopy on $\mathrm{K}^{-2}, \mathrm{~K}^{-1} \mathrm{~L}^{-1}$, and $\mathrm{L}^{-2}$ double core hole states of $\operatorname{SiX}_{4}\left(\mathrm{X}=\mathrm{H}, \mathrm{F}, \mathrm{Cl}\right.$, and $\left.\mathrm{CH}_{3}\right)$ molecules," Chem. Phys. 384, 28-35 (2011).

${ }^{43}$ O. Takahashi, N. V. Kryzhevoi, and K. Ueda, "Probing chemical environment with molecular double core-hole electron spectroscopy," J. Elec. Spectr. Rel. Phenom. 204, 290-302 
(2015), gas phase spectroscopic and dynamical studies at Free-Electron Lasers and other short wavelength sources.

${ }^{44}$ O. Takahashi, M. Tashiro, M. Ehara, K. Yamasaki, and K. Ueda, "Theoretical molecular double-core-hole spectroscopy of nucleobases," J. Phys. Chem. A 115, 12070-12082 (2011).

${ }^{45}$ N. V. Kryzhevoi, R. Santra, and L. S. Cederbaum, "Inner-shell single and double ionization potentials of aminophenol isomers," J. Chem. Phys. 135, 084302 (2011).

${ }^{46}$ W. Hua, K. Bennett, Y. Zhang, Y. Luo, and S. Mukamel, "Study of double core hole excitations in molecules by x-ray double-quantum-coherence signals: a multi-configuration simulation," Chem. Sci. 7, 5922-5933 (2016).

${ }^{47}$ M. G. Delcey, L. K. Sørensen, M. Vacher, R. C. Couto, and M. Lundberg, "Efficient calculations of a large number of highly excited states for multiconfigurational wavefunctions," J. Comput. Chem. 40, 1789-1799 (2019).

${ }^{48}$ X. Zheng, J. Liu, G. Doumy, L. Young, and L. Cheng, "Hetero-site double core ionization energies with sub-electronvolt accuracy from delta-coupled-cluster calculations," J. Phys. Chem. A 124, 4413-4426 (2020).

${ }^{49}$ J. Lee, D. W. Small, and M. Head-Gordon, "Excited states via coupled cluster theory without equation-of-motion methods: Seeking higher roots with application to doubly excited states and double core hole states," J. Chem. Phys. 151, 214103 (2019).

${ }^{50}$ R. Santra, N. V. Kryzhevoi, and L. S. Cederbaum, "X-ray two-photon photoelectron spectroscopy: A theoretical study of inner-shell spectra of the organic para-aminophenol molecule," Phys. Rev. Lett. 103, 013002 (2009).

${ }^{51}$ E. M. L. Ohrendorf, L. S. Cederbaum, and F. Tarantelli, "Double vacancies in the cores of silane and tetrafluorosilane," Phys. Rev. A 44, 205-217 (1991).

${ }^{52}$ M. Tashiro, K. Ueda, and M. Ehara, "Double core-hole correlation satellite spectra of $\mathrm{N}_{2}$ and CO molecules," Chem. Phys. Lett. 521, 45-51 (2012).

${ }^{53}$ S. Evangelisti, J.-P. Daudey, and J.-P. Malrieu, "Convergence of an improved CIPSI algorithm," Chem. Phys. 75, 91-102 (1983).

${ }^{54}$ R. J. Harrison, "Approximating full configuration interaction with selected configuration interaction and perturbation theory," J. Chem. Phys. 94, 5021-5031 (1991).

${ }^{55}$ K. Andersson, P.-Å. Malmqvist, B. O. Roos, A. J. Sadlej, and K. Wolinski, "Secondorder perturbation theory with a casscf reference function," J. Phys. Chem. 94, 5483-5488 (1990). 
${ }^{56}$ K. Andersson, P. Malmqvist, and B. O. Roos, "Second-order perturbation theory with a complete active space self-consistent field reference function," J. Chem. Phys. 96, 12181226 (1992).

${ }^{57}$ P.-A. Malmqvist, K. Pierloot, A. R. M. Shahi, C. J. Cramer, and L. Gagliardi, "The restricted active space followed by second-order perturbation theory method: Theory and application to the study of $\mathrm{CuO}_{2}$ and $\mathrm{Cu}_{2} \mathrm{O}_{2}$ systems," J. Chem. Phys. 128, 204109 (2008).

${ }^{58}$ V. Sauri, L. Serrano-Andrés, A. R. M. Shahi, L. Gagliardi, S. Vancoillie, and K. Pierloot, "Multiconfigurational second-order perturbation theory restricted active space (raspt2) method for electronic excited states: A benchmark study," J. Chem. Theory Comput. 7, 153-168 (2011).

${ }^{59}$ P.-A. Malmqvist and B. O. Roos, "The CASSCF state interaction method," Chem. Phys. Lett. 155, 189-194 (1989).

${ }^{60} \mathrm{~S}$. F. Boys, "Construction of some molecular orbitals to be approximately invariant for changes from one molecule to another," Rev. Mod. Phys. 32, 296-299 (1960).

${ }^{61}$ J. Pipek and P. G. Mezey, "A fast intrinsic localization procedure applicable for ab initio and semiempirical linear combination of atomic orbital wave functions," The Journal of Chemical Physics 90, 4916-4926 (1989).

${ }^{62}$ F. Aquilante, T. B. Pedersen, A. Sánchez de Merás, and H. Koch, "Fast noniterative orbital localization for large molecules," J. Chem. Phys. 125, 174101 (2006).

${ }^{63}$ T. Moitra, A. Ponzi, H. Koch, S. Coriani, and P. Decleva, "Accurate description of photoionization dynamical parameters," J. Phys. Chem. Lett. 11, 5330-5337 (2020), https://doi.org/10.1021/acs.jpclett.0c01337.

${ }^{64}$ T. Moitra, S. Coriani, and P. Decleva, "Capturing correlation effects on photoionization dynamics," J. Chem. Theory Comput. 17, 5064-5079 (2021), https://doi.org/10.1021/acs.jctc.1c00303.

${ }^{65} \mathrm{P}$. A. Malmqvist, "Calculation of transition density matrices by nonunitary orbital transformations," Int. J. Quantum Chem. 30, 479-494 (1986).

${ }^{66}$ G. Grell, S. I. Bokarev, B. Winter, R. Seidel, E. F. Aziz, S. G. Aziz, and O. Kühn, "Multi-reference approach to the calculation of photoelectron spectra including spin-orbit coupling," J. Chem. Phys. 143, 074104 (2015).

${ }^{67}$ G. Grell, O. Kühn, and S. I. Bokarev, "Multireference quantum chemistry protocol for simulating autoionization spectra: Test of ionization continuum models for the neon atom," 
Phys. Rev. A 100, 042512 (2019).

${ }^{68}$ G. Grell and S. I. Bokarev, "Multi-reference protocol for (auto)ionization spectra: Application to molecules," J. Chem. Phys. 152, 074108 (2020).

${ }^{69}$ C. D. Sherrill and H. F. Schaefer, "The configuration interaction method: Advances in highly correlated approaches," (Academic Press, 1999) pp. 143-269.

${ }^{70}$ I. Fdez. Galván, M. Vacher, A. Alavi, C. Angeli, F. Aquilante, J. Autschbach, J. J. Bao, S. I. Bokarev, N. A. Bogdanov, R. K. Carlson, L. F. Chibotaru, J. Creutzberg, N. Dattani, M. G. Delcey, S. S. Dong, A. Dreuw, L. Freitag, L. M. Frutos, L. Gagliardi, F. Gendron, A. Giussani, L. González, G. Grell, M. Guo, C. E. Hoyer, M. Johansson, S. Keller, S. Knecht, G. Kovačević, E. Källman, G. Li Manni, M. Lundberg, Y. Ma, S. Mai, J. P. Malhado, P.-A. Malmqvist, P. Marquetand, S. A. Mewes, J. Norell, M. Olivucci, M. Oppel, Q. M. Phung, K. Pierloot, F. Plasser, M. Reiher, A. M. Sand, I. Schapiro, P. Sharma, C. J. Stein, L. K. Sørensen, D. G. Truhlar, M. Ugandi, L. Ungur, A. Valentini, S. Vancoillie, V. Veryazov, O. Weser, T. A. Wesołowski, P.-O. Widmark, S. Wouters, A. Zech, J. P. Zobel, and R. Lindh, "Openmolcas: From source code to insight," J. Chem. Theory Comput. 15, 5925-5964 (2019).

${ }^{71}$ D. E. Woon and T. H. Dunning, "Gaussian basis sets for use in correlated molecular calculations. iv. calculation of static electrical response properties," J. Chem. Phys. 100, 2975-2988 (1994).

${ }^{72}$ N. B. Balabanov and K. A. Peterson, "Systematically convergent basis sets for transition metals. I. All-electron correlation consistent basis sets for the 3d elements Sc-Zn," J. Chem. Phys. 123, 064107 (2005).

${ }^{73}$ T. Nakajima and K. Hirao, "The Douglas-Kroll-Hess Approach," Chem. Rev. 112, 385$402(2012)$.

74“NIST Chemistry WebBook - Standard Reference Database Number 69," (2021).

${ }^{75}$ A. Rohatgi, "Webplotdigitizer," (2020).

${ }^{76}$ T. Åberg, "Theory of x-ray satellites," Phys. Rev. 156, 35-41 (1967). 